\title{
Self-optimizing control: the missing link between steady-state optimization and control
}

\author{
Sigurd Skogestad * \\ Department of Chemical Engineering, Norwegian University of Science and Technology, $N-7491$ Trondheim, Norway
}

\begin{abstract}
Real-time steady-state optimization (RTO) has become increasing popular in recent years. But how should this optimal policy be implemented in the control system? It is argued that the goal is to find a set of controlled variables which, when kept at constant setpoints, indirectly lead to near-optimal operation with acceptable loss. This is denoted 'self-optimizing' control. C 2000 Elsevier Science Ltd. All rights reserved.
\end{abstract}

Keywords: Chemical process control; Control structure design; Plantwide control

\section{Introduction}

If we consider the control system in a chemical plant, then we find that it is structured hierarchically into several layers, each operating on a different time scale. Typically, layers include scheduling (weeks), site-wide (real-time) optimization (day), local optimization (h), supervisory/predictive control (min) and stabilizing and regulatory control (s); see Fig. 1. The layers are interconnected through the controlled variable $c$, whereby the upper layer computes the setpoint value $c_{\mathrm{s}}$ to be implemented by the lower layer. We usually assume timc-scale separation, which for our purposes implies that, the setpoints $c_{\mathrm{s}}$ can assumed to be immediately implemented by the layers below. Which should these internal controlled variable $c$ be? That is, what should we control? This paper attempts to answer this question.

More generally, the issue of selecting controlled variables is the first subtask in the plantwide control or control structure design problem (Foss, 1973; Morari, 1982; Skogestad \& Postlethwaite, 1996):

1. selection of controlled variable $c$;

2. selection of manipulated variable $m$;

3. selection of measurements $v$ (for control purposes including stabilization);

\footnotetext{
* Corresponding author. Tel.: +47-7359-4154; fax: + 47-73594080 .

E-mail address: skoge@chembio.ntnu.no (S. Skogestad)
}

4. selection of a control configuration (structure of the controller that interconnects measurements/setpoints and manipulated variables);

5. selection of controller type (control low specification, e.g. PID, decoupler, LQG, etc.).

Even though control engineering is well developed in terms of providing optimal control algorithms, it is clear that must of the existing theories provide little help when it comes to making the above structural decisions.

\section{Previous work}

Inspired by the work of Findeisen, Bailey, Brdys, Malinowski, Tatjewski and Wozniak (1980), the basic idea of self-optimizing control was formulated about 20 years ago by Morari, Stephanopoulos and Arkun (1980). Morari et al. (1980) wrote that 'in attempting to synthesize a feedback optimizing control structure, our main objective is to translate the economic objectives into process control objectives. In other words, we want to find a function $c$ of the process variables which when held constant, leads automatically to the optimal adjustments of the manipulated variables, and with it, the optimal operating conditions. [...] This means that by keeping the function $c(u, d)$ at the setpoint $c_{\mathrm{s}}$, through the use of the manipulated variable $u$, for various disturbances $d$, it follows uniquely that the process is 
operating at the optimal steady-state'. If we replace $i$ the term 'optimal adjustments' by 'acceptable adjustments (in terms of the loss)' then the above is a precise description of what we in this paper denote a self-optimizing control structure. The only factor they fail to consider is the effect of implementation error $c-c_{\mathrm{s}}$. Somewhat surprisingly, the idea of 'feedback optimizing control' of Morari et al. (1980) has up to now received very little attention. One reason is probably that the paper also dealt with the issue of finding the optimal operation (and not only on how to implement it), and another reason is that the only example in the paper happened to result in an implementation with the controlled variables at their constraints. The constrained case is 'easy' from an implementation point of view, because the simplest and optimal implementation is to simply maintain the constrained variables at their constraints ('active constraint control').

At about the same time, Shinnar (1981) published a more intuitive process-oriented approach for selecting controlled variables, and applied it to the control of a fluidized catalytic cracker (FCC). The work may at first seem unrelated but if one translates the words and notation, then one realizes that Shinnar's ideas are close to the ideas presented in this paper and in Morari et al.

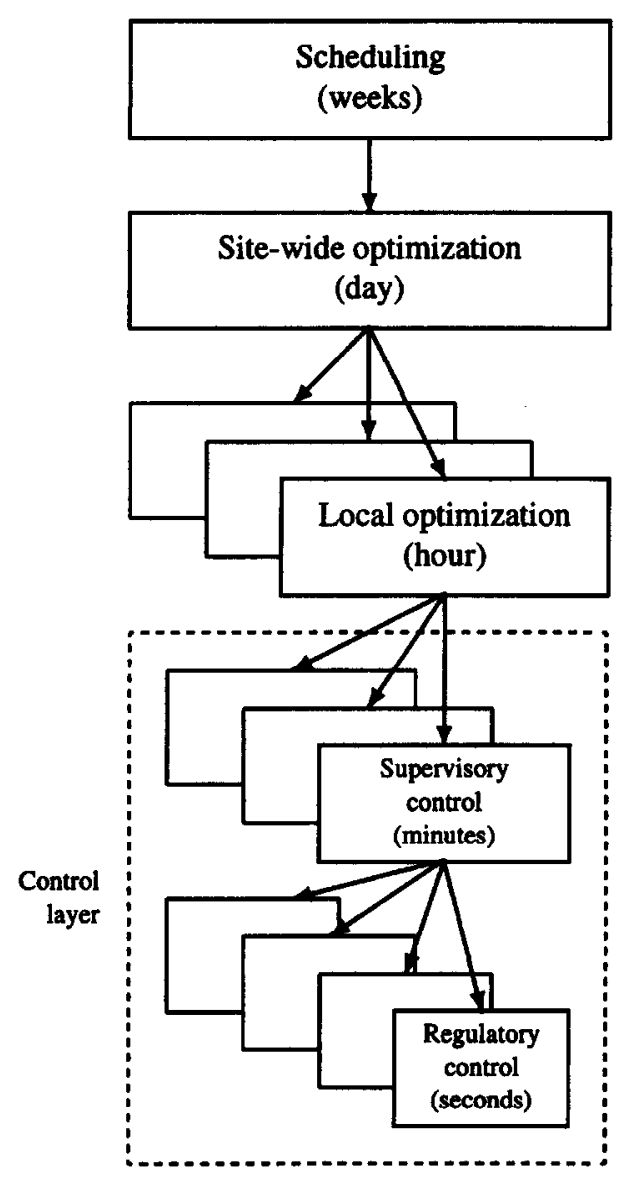

Fig. 1. Typical control hierarchy' in a chemical plant.
(1980). The similar later paper by Arbel, Rinard and Shinnar (1996) extended the FCC case study, and introduced the concepts of 'dominant variables' and 'partial control'. Tyreus (1999) provides some interesting ideas on how to select dominant variables.

Luyben (1988) introduced the term 'eigenstructure' to describe the inherently best control structure (with the best self-regulating and self-optimizing property). However, he did not really define the term, and also the name is unfortunate since 'eigenstructure' has another unrelated mathematical meaning in terms of eigenvalues. Fisher, Doherty and Douglas (1988) discuss selection of controlled variables, mainly focused towards active constraint control. However, somewhat hidden in their HDA example (p. 614) one finds statements about selecting controlled variables which optimal values are insensitive to disturbances. Narraway and Perkins (1993, 1994), Narraway, Perkins and Barton (1991) strongly stress the need to base the selection of the control structure on economics, and they discuss the effect of disturbances on the economics. However, they do not formulate any rules or procedures for selecting controlled variables. In his book Rijnsdorp (1991) gives on page 99 a stepwise design procedure for designing optimizing control systems for process units. One step is to 'transfer the result into on-line algorithms for adjusting the degrees of freedom for optimization'. Marlin and Hrymak (1997) and Mizoguchi, Marlin and Hrymak (1995) stress the need to find a good way of implementing the optimal solution in terms how the control system should respond to disturbances, 'i.e. the key constraints to remain active, variables to be maximized, priority for adjusting manipulated variables, and so forth'. Finally, Zheng, Mahajannam and Douglas (1999) present a procedure for selecting controlled variables based on economic penalties that is similar to the approach presented in this paper (apparently, the work has been performed independently), but they also do not consider the implementation error.

\section{Optimal operation and its implementation}

When controlling a chemical plant our first concern is to stabilize the plant and keep its operation within given constraints. These issues may consume some degrees of freedom (e.g. to stabilize levels with no steadystate effect and to satisfy 'active' product specifications), but there will generally be many degrees of freedom $u$ left. What should these be used for?

Loosely speaking, they should be used to 'optimize the operation'. There may be many issues involved, and to trade them off against each other in a systematic manner we usually quantify a scalar performance (cost) index $J$, which should be minimized. In many cases this index is an economic measure, e.g. the operation cost. For example, $J$ could be of the form 


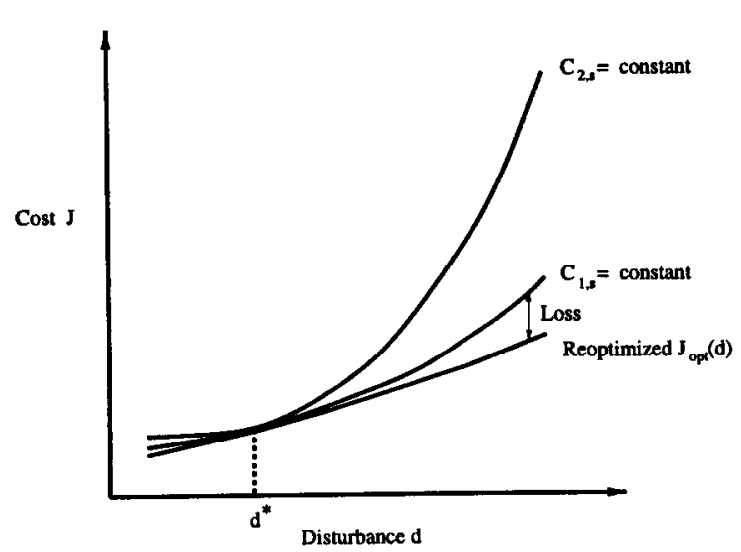

Fig. 2. Loss imposed by keeping constant setpoint for the controlled variable.

$J=J_{u}(u, d)=\int_{0}^{T} \phi(u, d) \mathrm{d} t$

where, $u$ are the degrees of freedom for optimization, $d$ are time-varying disturbances, and $T$ is the total operation time. The optimizing control problem may then be formulated as

$\min _{u} J_{u}(u, d)$

subject to the inequality constraints

$g(u, d) \leq 0$

where, $u$ are the independent variables we can affect (degrees of freedom), and $d$ are independent variables we cannot affect (disturbances). In this paper we will for simplicity use steady-state mociels and the integration in Eq. (1) may be replaced by time-averaging over the various steady-states. The main justification for using a steady-state analysis is that the economic performance is primarily determined by steady-state considerations. The effect of the dynamic control performance can be partly included in the economic analysis by introducing a control error term as an additional disturbance.

There are two main issues when it comes to optimizing control. The first is the mathematical and numerical problem of solving the optimization in Eq. (2) to obtain the optimal operating point. The optimization problem may be very large, with hundreds of thousands of equations and hundreds of degree of freedom (e.g. for a complete ethylene plant), but with today's computers and optimization methods this problem is solvable, and it is indeed solved routinely today in some plants. The second issue, the focus of this paper is how the optimal solution should be implemented in practice, that is, which variable $c$ should be held constant between each reoptimization. Surprisingly, this issue has received much less attention.

\section{Self-optimizing control}

The method advocated in this paper for selecting controlled variables (task 1) follows the ideas of Skogestad and Postlethwaite (1996) and Morari et al. (1980) is very simple. The basis is to define mathematically the quality of operation in terms of a scalar cost function $J$ to be minimized. To achieve truly optimal operation we would need a perfect model, we would need to measure all disturbances, and we would need to solve the resulting dynamic optimization problem online. This is unrealistic in most cases, and the question is if it is possible to find a simpler implementation, which still operates satisfactorily (with an acceptable loss). More precisely, the loss $L$ is defined as the difference between the actual value of the cost function obtained with a specific control strategy, and the truly optimal value of the cost function, i.e.

$L=J-J_{\text {opt }}$

The simplest operation would result if we could select controlled variables such that we obtained acceptable operation with constant setpoints, thus effectively turning the complex optimization problem into a simple feedback problem and achieve what we here call 'selfoptimizing control':

Self-optimizing control is when we can achieve an acceptable loss $L$ with constant setpoint values $c_{s}$ for the controlled variables.

(The reader is probably familiar with the term selfregulation, which is when acceptable dynamic control performance can be obtained with constant manipulated inputs. Self-optimizing control is a direct generalization to the case where we can achieve acceptable (economic) performance with constant controlled variables.)

The idea is that by locally controlling the right variable $c$, we can take care of most of the disturbances, and thus reduce the need for continuous reoptimization. This also reduces the need for model information and tends to make the implementation more robust. On the other hand, it usually implies a performance loss compared with the 'true' optimal (centralized) solution, and the challenge is to find a 'self-optimizing' control structure (i.e. to find the right controlled variable $c$ ) for which the loss $L$ is acceptable.

A well-known example of self-optimizing control is the 'cake baking process', where the operation is indirectly kept close to its optimum by controlling the oven temperature at the setpoint given in the cook book (which is this case is the 'optimizer').

The idea is further illustrated in Fig. 2, where we see that there is a loss if we keep a constant setpoint rather 
than reoptimizing when a disturbance moves the process away from its nominally optimal operating point (denoted ${ }^{*}$ ). For the case illustrated in the figure it is better (with a smaller loss) to keep the setpoint $c_{1 s}$ constant than to keep $c_{2 s}$ constant.

An additional concern with the constant setpoint policy is that there will always be an implementation error $d_{\mathrm{c}}=c-c_{\mathrm{s}}$, e.g. caused by measurement error. The implementation error may cause a large additional loss if the optimum surface is 'sharp'. To be more specific, we may, as illustrated in Fig. 3 distinguish between three classes of problems when it comes to the actual implementation:

(a) Constrained optimum: implementation easy. In the figure it is shown the case where the minimum value of the cost $J$ is obtained for $c=c_{\text {min }}$. In this case there is no loss imposed by keeping a constant $c_{\mathrm{s}}=$ $c_{\text {min }}$. In addition, implementation of an 'active' constraint is usually easy; e.g. it is easy to keep a valve closed.

(b) Unconstrained flat optimum: implementation easy. In this case the cost is insensitive to value of the

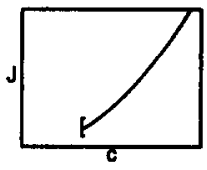

(a)

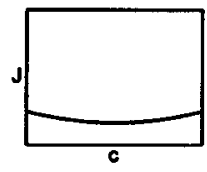

(b)

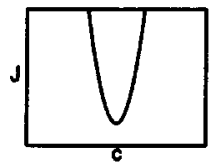

(c)
Fig. 3. Implementing the controlled variable.

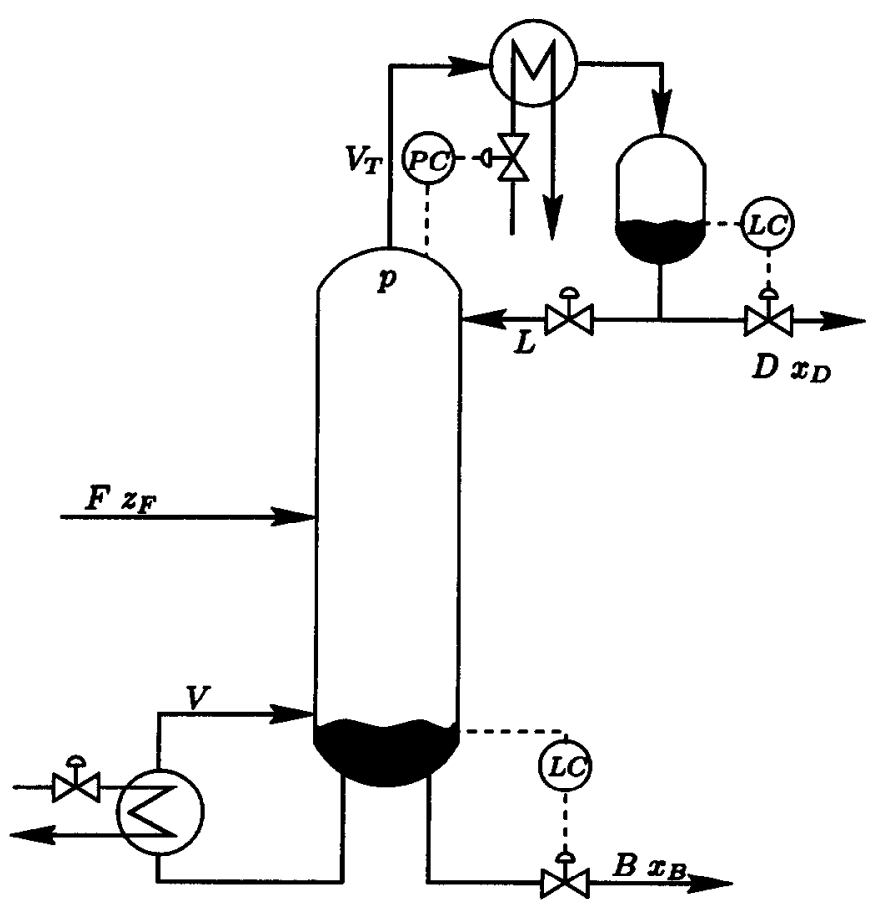

Fig. 4. Typical distillation column controlled with the $L V$-configuration. controlled variable $c$, and implementation is again easy.

(c) Unconstrained sharp optimum: implementation difficult. The more difficult problems for implementation is when the cost (operation) is sensitive to value of the controlled variable $c$. In this case, we want to find another controlled variable $c$ in which the optimum is flatter.

\section{Requirements for controlled variables}

We can derive the following four requirements for a good candidate controlled $c$ variable (also see Skogestad \& Postlethwaite (1996), page 404):

Requirement 1. Its optimal value is insensitive to disturbances (so that the setpoint error is small).

Requirement 2. It is easy to measure and control accurately (so that the implementation error is small). Requirement 3. Its value is sensitive to changes in the manipulated variable $u$; that is, the gain from $u$ to $y$ is large (so that even a large error in controlled variable $c$ results in only a small error in $u$ ). Equivalently, the optimum should be 'flat' with respect to the variable $c$.

Requirement 4. For cases with two or more controlled variables, the selected variables should not be closely correlated.

All four requirements should be satisfied. For example, assume that we have a mixture of three components, and we have a measurement of the sum of the three mole fractions, $c=x_{\mathrm{A}}+x_{\mathrm{B}}+x_{\mathrm{C}}$. This measurement is always 1 and thus independent of disturbances (so requirement 1 is satisfied), but it is of course not a suitably controlled variable because it is also insensitive to the manipulated variable $u$ (so requirement 3 is not satisfied). Requirement 3 also eliminates variables that have an extremum (maximum or minimum) when the cost has its minimum, because the gain is 0 for such variables.

\section{Distillation example}

To give the reader some appreciation of the issues, we consider a distillation plant where the plant economics are mainly determined by its steady-state behavior. With a given feed stream and a specified column pressure, a conventional two-product distillation column, as shown in Fig. 4, has two degrees of freedom at steady-state $\left(N_{\mathrm{opt}}=N_{u}=2\right)$. (From a control point of view the column has $N_{\mathrm{m}}=5$ degrees of freedom, but two degrees of freedom are needed to stabilize the reboiler and condenser holdups, which have no steady- 
Table 1

Losses in $\$ / \min$ for various disturbances

\begin{tabular}{|c|c|c|c|c|}
\hline & $x_{\mathrm{B}}=0.04$ & $D / F=0.639$ & $L / F=15.065$ & $L / D=23.57$ \\
\hline$F: 1 \rightarrow 1.3$ & 0 & 0 & 0 & 0 \\
\hline$z_{F}: 0.65 \rightarrow 0.75$ & 0.019 & 2.530 & 0.006 & 0.129 \\
\hline$q_{\mathrm{F}}: 1 \rightarrow 0.5$ & 0.000 & 0.000 & 0.001 & 0.000 \\
\hline $\begin{array}{l}20 \% \text { Imple- } \\
\text { mentation error }\end{array}$ & 0.012 & Inf. $^{a}$ & 0.119 & 0.130 \\
\hline
\end{tabular}

${ }^{a}$ Inf. denotes infeasible operation.

state effect, and one degree of freedom is used to control the pressure at its given value). The two steady-state degrees of freedom, e.g. selected to be the reflux flow $L$ and the distillate flow $D$,

$u=\left[\begin{array}{l}L \\ D\end{array}\right]$

(this is not a unique choice) may be used to optimize the operation of the plant. However the question is, how should the optimal solution be implemented, that is which two variables $c$ should be specified and controlled during operation?

To answer this question in a quantitative manner, we need to define the constraints for the operation and the cost function $J$ to be minimized.

Constraints. We assume that the mole fraction of light component in the distillate product $x_{\mathrm{D}}$ must be above 0.95 , and that to avoid flooding the capacity of the column is limited by a maximum allowed vapor load.

$x_{\mathrm{D}} \geq x_{\mathrm{D}, \min }=0.95 ; \quad V \leq V_{\max }$

Cost function. Rather than minimizing the cost $J$, it is more natural in this case to maximize the profit $P=-J$, which is the product value minus the feed costs and the operational (energy) costs which are proportional to the vapor flow $V$,

$P=p_{\mathrm{D}} D+p_{\mathrm{B}} B-p_{\mathrm{F}} F-p_{\mathrm{V}} V$

\subsection{Case 1: constrained operation}

Let us first consider a case where

- distillate is the more valuable product $\left(p_{\mathrm{D}} \gg p_{\mathrm{B}}\right)$,

- energy costs are low ( $p_{\mathrm{V}}$ is small).

In this case, it is optimal to operate the column at maximum load (to reduce the loss of light component in the bottom) and with the distillate composition at its specification (to maximize distillate flow by including as much heavy component as possible) (Gordon, 1986), i.e.
$V_{\mathrm{opt}}=V_{\max } ; \quad x_{\mathrm{D}, \mathrm{opt}}=x_{\mathrm{D}, \min }=0.95$

Thus, the optimum lies at constraints and implementation is obvious: we should select the vapor rate $V$ and the distillate composition $x_{\mathrm{D}}$ as the controlled variables,

$c=\left[\begin{array}{c}V \\ x_{\mathrm{D}}\end{array}\right] ; \quad c_{\mathrm{s}}=\left[\begin{array}{c}V_{\max } \\ x_{\mathrm{D}, \min }\end{array}\right]$

In practice, we may implement this using a lowerlevel feedback control system where we adjust the boilup $V$ to keep the pressure drop over the column (an indicator of flooding, i.e. $V_{\max }$ ) below a certain limit, and adjust the reflux $L$ (or some other flow, depending on how the level and pressure control system is configured) so that $x_{\mathrm{D}}$ is kept constant.

\subsection{Case 2: partly constrained operation}

Next, consider a case where

- distillate is the more valuable product $\left(p_{\mathrm{D}}>p_{\mathrm{B}}\right)$,

- energy costs are relatively high (such that the term $p_{V} V$ contributes significantly to $J$ ).

Also in this case it is optimal to have the distillate composition at its specification, $x_{\mathrm{D}}=x_{\mathrm{D}, \min }$, but to save energy we will have $V<V_{\max }$. Again, we should select $x_{\mathrm{D}}$ as a controlled variable, but it is not clear what the other variable should be. Some options may be $x_{\mathrm{B}}, L, V, D, L / F$ or $L / D$. To find the best choice a more detailed choice is needed, for example, based on evaluating the economic loss for various disturbances. We often find that the purity $x_{\mathrm{B}}$ is a good choice, but 'two-point' composition control is generally to be avoided because of poor dynamic performance.

The losses for a propane-propylene column case study (Skogestad, 2000) are given in Table 1. We see that keeping $L / F$ constant is a good alternative for 'self-optimizing' control with a loss similar to that of keeping $x_{\mathrm{B}}$ constant. However, it is not a good choice to keep $D / F$ or $L / D$ constant.

\subsection{Case 3: unconstrained operation}

For a case with

- bottoms product is the more valuable product $\left(p_{\mathrm{B}}>p_{\mathrm{D}}\right)$, but its price $p_{\mathrm{B}}$ is reduced as it gets impure,

- energy costs are relatively high,

the optimum may be unconstrained in both variables that is,

$V_{\text {opt }}<V_{\text {max }} ; \quad x_{\mathrm{D}, \text { opt }}>x_{\mathrm{D}, \min }$

Implementation in this case is again not obvious. Some candidate sets of controlled variable $c$ are 


$$
\begin{gathered}
c_{1}=\left[\begin{array}{c}
x_{\mathrm{D}} \\
x_{\mathrm{B}}
\end{array}\right] ; \quad c_{2}=\left[\begin{array}{c}
T_{\mathrm{top}} \\
T_{\mathrm{btm}}
\end{array}\right] ; \quad c_{3}=\left[\begin{array}{c}
x_{\mathrm{D}} \\
V
\end{array}\right] ; \quad c_{4}=\left[\begin{array}{c}
L \\
V
\end{array}\right] \\
c_{5}=\left[\begin{array}{c}
L / D \\
V / B
\end{array}\right]
\end{gathered}
$$

and there are many others. Controlled variables $c_{1}$ and $c_{2}$ will yield a 'two-point' control system where we close two loops for quality control; $c_{3}$ yields a 'one-point' control system where only one quality loop is closed; whereas $c_{4}$ and $c_{5}$ are 'open-loop' policies which require no additional feedback loops (except for the level and pressure loops already mentioned). All of these choices of controlled variables will have different self-optimizing control properties and a more careful analysis is required.

\subsection{Case 4: feed rate as degree of freedom}

Let us go back to case 1 , but assume now that the feed rate is a degree of freedom. Again we will find that the capacity and purity constraints are active,

$V_{\mathrm{opt}}=V_{\text {max }} ; \quad x_{\mathrm{D}, \mathrm{opt}}=x_{\mathrm{D}, \min }=0.95$

but since we will attempt to push more feed through the column the operation will nevertheless be different from case 1 . We will get a less pure bottom product and thus a larger loss of the valuable light component.

Let us consider this in some more detail. In case 1 the objective of optimizing the profit $P$ is equivalent to maximizing $P / F$ (since $F$ is given). On the other hand, in case 4 the feed rate is a degree of freedom and at the optimum we have $\partial P / \partial F=0$, and the resulting value of $P / F$ will obviously be smaller than in case 1 .

\section{A fundamental difference between design and operation}

The last finding has some interesting implications regarding the difference between design and operation. In both cases we want to maximize the profit (value increase) $P$. During design we always assume a fixed feed rate and thus maximize $P / F$. On the other hand, during operation the feed rate is often a free variable, and as shown above we will get that

$\left(\frac{P}{F}\right)_{\text {operation }}<\left(\frac{P}{F}\right)_{\text {design }}$

Thus, if we optimize the operation we will use the raw materials and energy less effectively than we designed for. An obvious question is, is this an ethical and environmental dilemma?

\section{Conclusion}

The results from the steady-state optimization are implemented by computing the setpoints of the controlled variable $c$. A set of controlled variables is denoted self-optimizing if we can achieve an acceptable (economic) loss with constant setpoints $c_{\mathrm{s}}$. Important steps in evaluating self-optimizing control are degrees of freedom analysis, definition of optimal operation (cost and constraints), and evaluation of the loss for the set of disturbances.

\section{Acknowledgements}

The ideas in this paper have developed gradually over several years. Valuable discussions with and contributions from J.C. Morud, B. Glemmestad, I.J. Halvorsen, K. Havre, T. Larsson, and M.S. Govatsmark are gratefully acknowledged.

\section{References}

Arbel, A., Rinard, I. H., \& Shinnar, R. (1996). Dynamics and control of fluidized catalytic crackers. 3 designing the control system: choice of manipulated and measured variables for partial control. Industrial Engineering \& Chemical Research, 35(7), 2215-2233.

Findeisen, W., Bailey, F. N., Brdys, M., Malinowski, K., Tatjewski, P., \& Wozniak, A. (1980). Control and coordination in hierarchical systems. New York: Wiley.

Fisher, W. R., Doherty, M. F., \& Douglas, J. M. (1988). The interface between design and control. 3: selecting a set of controlled variables. Industrial Engineering \& Chemical Research, 27(4), 611-615.

Foss, C. S. (1973). Critique of chemical process control theory. American Institute of Chemical Engineering Journal, 19(2), 209214.

Gordon, M. L. (1986). Simple optimization for dual composition control. Hydrocarbon Processing, June 1986, 59-62.

Luyben, W. L. (1988). The concept of eigenstructure in process control. Industrial Engineering \& Chemical Research, 27(1), 206208.

Marlin, T. E., \& Hrymak, A. N. (1997). Real-time operations optimization of continuous processes. In: Fifth International Conference on Chemical Process Control (CPC-5) (pp. 156-164). American Institute of Chemical Engineering Journal Symposium Series, vol. 9. Lake Tahoe.

Mizoguchi, A., Marlin, T. E., \& Hrymak, A. N. (1995). Operations optimization and control design for a petroleum distillation process. Canadian Journal of Chemical Engineering, 73, 896-907.

Morari, M. (1982). Integrated plant control: a solution at hand or a research topic for the next decade? In: Proceedings of of Second International Conference on Chemical Process Control (CPC-2) (pp. 467-495). Sea Island, Georgia.

Morari, M., Stephanopoulos, G., \& Arkun, Y. (1980). Studies in the synthesis of control structures for chemical processes. Part 1: formulation of the problem. Process decomposition and the classification of the control task. Analysis of the optimizing control structures. American Institute of Chemical Engineering Journal, $26(2), 220-232$. 
Narraway, L. T., \& Perkins, J. D. (1993). Selection of process control structure based on linear dynamic economics. Industrial Engineering \& Chemical Research, 32(11), 2681-2692.

Narraway, L., \& Perkins, J. (1994). Selection of process control structures based on economics. Computers \& Chemical Engineering, 18(Suppl.), S511-S515.

Narraway, L. T., Perkins, J. D., \& Barton, G. W. (1991). Interaction between process design and process control: economic analysis of process dynamics. Journal of Process Control, 1, 243-250.

Rijnsdorp, J. E. (1991). Integrated process control and automation. Amsterdam: Elsevier.
Shinnar, R. (1981). Chemical reactor modelling for purposes of controller design. Cheng. Engineering Communication, 9, 73-99.

Skogestad, S. (2000). Plantwide control: the search for the self-optimizing control structure. Journal of Process Control, 10, in press.

Skogestad, S., \& Postlethwaite, I. (1996). Multivariable feedback control. New York: Wiley.

Tyreus, B. D. (1999). Dominant variables for partial control; part 1 and 2. Industria

l Engineering \& Chemical Research, 38, 1432-1455.

Zheng, A., Mahajannam, R. V., \& Douglas, J. M. (1999). Hierarchical procedure for plantwide control system synthesis. American Institute of Chemical Engineering Journal, 45, 1255-1265. 